\title{
Factor V Leiden Thrombophilia Causing Recurrent Thrombosis
}

Md. Atiquzzaman ${ }^{1 *}$, A B M Sarwar-e-Alam², Muhammad Mazharul Hoque ${ }^{3}$, Md. Motiul Islam4, Tarikul

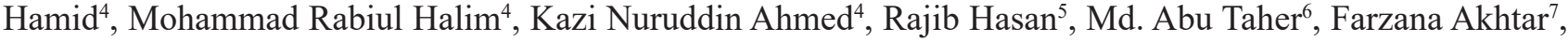
Md. Ashikuzzaman Sohan ${ }^{8}$

DOI: https://doi.org/10.3329/bccj.v9i2.56158

\begin{abstract}
:
Normal hemostasis requires a delicate balance between the natural procoagulant and anticoagulant systems. Thrombophilia is an abnormality of the coagulation or fibrinolytic system that results in a hypercoagulable state and increases the risk for thrombotic event by forming intravascular thrombus. The predisposition to form such blood clot may be inherited or acquired. In inherited thrombophilia, there is deficiency of natural anti-coagulants, e.g. anti-thrombin III, protein $C$ and $S$ or increase formation of human coagulant factors, e.g., factor $V$ Leiden or prothrombin gene 20210A. Factor V Leiden thrombophilia is extremely rare in Bangladesh.

We report this unique case of a young lady of 46-year-old presented with continuous headache and found to have extensive cerebral venous thrombosis with bilateral $6^{\text {th }}$ cranial nerve palsy. She had suffered from acute arterial occlusion of left upper limb in September 2016, 2 years prior to current presentation. We suspect thrombophilia according to her presentation. The subsequent step is to screen for-human natural anti-coagulant factors deficiency or presence of mutated form of factor $V$ (factor $V$ Leiden) and prothrombin gene mutation. We screened for and found to have presence of factor $V$ Leiden in our patient. We always should remember that, thrombotic events are increasingly recognized as a significant source of mortality and morbidity, so screening should be considered in selected individuals if criteria suggests.
\end{abstract}

Keywords: Factor V Leiden, thrombophilia, recurrent thrombosis, APC resistance.

\section{Introduction:}

Thrombophilias represent inherited and acquired hypercoagulable states that increase the risk of venous and, in some cases, arterial thromboembolism. The prevalence varies by population. Heterozygosity for Factor V Leiden occurs in

1. Senior Clinical Staff, General ICU, Asgar Ali Hospital, Dhaka-1204, Bangladesh.

2. Senior Consultant, Medicine, Asgar Ali Hospital, Dhaka-1204, Bangladesh.

3. Clinical Staff, Intensive Care Unit, Asgar Ali Hospital, Dhaka-1204, Bangladesh.

4. Associate Consultant, Intensive Care Unit, Asgar Ali Hospital, Dhaka-1204, Bangladesh.

5. Specialist, Intensive Care Unit, Asgar Ali Hospital, Dhaka-1204, Bangladesh.

6. Assistant Registrar, Medicine, Jalalabad Ragib-Rabeya Medical College Hospital, Sylhet, Bangladesh.

7. Senior Medical Officer, Department of Obst. \& Gynae, OGSB Hospital \& Institute of Reproductive \& Child Health, Dhaka, Bangladesh.

8. Senior Resident Medical Officer, Intensive Care Unit, Asgar Ali Hospital, Dhaka-1204, Bangladesh.

\section{*Corresponding Author:}

Dr. Md. Atiquzzaman, MBBS

Senior Clinical Staff, Intensive Care Unit, Asgar Ali Hospital 111/1/A, Distillery Road, Gandaria, Dhaka-1204, Bangladesh. E mail: drazrinku@gmail.com

Contact: +8801711939036
$3-8 \%$ of the general US and European populations. The highest heterozygosity rate is found in Europe, but the mutation is almost non-existent in Southeast Asian, African, and indigenous Australian populations ${ }^{1,2,6}$. The relative risk for venous thromboembolism (VTE) is increased 3- to 8-fold in Factor $\mathrm{V}$ Leiden heterozygotes. Lower relative risks are reported in heterozygotes identified from general population screening. The thrombotic risk is increased 10- to 80 -fold in homozygotes. The risk for primary upper extremity thrombosis (not related to malignancy or a venous catheter) is increased 3- to 6-fold in Factor V Leiden heterozygotes ${ }^{2}$. A homozygous abnormality or combination of two or more heterozygous abnormal factors can lead to clinically apparent thrombotic disorders at an early age. However, milder heterozygous traits, when existing alone, are more often discovered by laboratory investigation ${ }^{3}$.

\section{CASE REPORT}

A 46-year-old hypertensive lady was admitted to the Asgar Ali Hospital, Dhaka, Bangladesh complaining of continuous headache and blurring of vision for last 15 days. She was well 15 days prior to this hospitalization. Then she developed global headache, which was band like and continuous but fluctuating in intensity over time, no radiation of pain towards neck or face. Patient scored her headache as 7/10 on severity scoring, which was disturbing her daily activities. She also experienced double vision on lateral gaze during last 2 weeks. There was no photophobia or phonophobia but she felt less alert than usual. She also had sleep disturbance during this 
time. Patient also felt nauseated during this period but no history of vomiting. No history of fever, rash, weight loss, altered level of consciousness, convulsion, head trauma. Also no history of cough, hemoptysis or recent contact with tuberculosis patient. No history suggestive of sinusitis. Her previous medical history revealed, acute left upper limb arterial occultation and for which embolectomy done on September 2016 at National Institute of Cardio-Vascular Diseases (NICVD), Dhaka, Bangladesh and the limb was saved. But afterwards she was not taking any blood thinner. A review duplex study of left upper limb was done on 2017 which showed no Doppler evidence of thrombus in left upper limb vascular channels. None of her family members had such type of illness. Her obstetric history was uneventful, no history of abortion or miscarriage. She has only one child. Apart from paracetamol, patient didn't take any analgesic for this problem. She was hypertensive, her blood pressure was well controlled with medication. She had no history of taking oral contraceptive pills (OCP), any anti-platelet or anti-coagulant drugs, any herbal remedies or over the counter drugs. Patient was non-smoker \& non-alcoholic. No history of betel nut chewing or using any recreational drugs. General examination revealed no abnormalities apart from embolectomy scar on left upper limb (Figure 2). All her vital signs were normal. Nervous system examination revealed, Glasgow Coma Scale score of 15, Pupil $2.5 \mathrm{~mm}$, bilaterally equally reacting to bright light, bilateral $6^{\text {th }}$ cranial nerve palsy (Figure 1). There was no evidence of any focal neurological deficit or meningism. Fundoscopy revealed bilateral papilloedema with single flame shaped hemorrhage at left eye. Other systemic examinations revealed no abnormalities. Laboratory evaluation revealed platelet count $500 \mathrm{~K} / \mu \mathrm{L}$, there was thrombocytosis in peripheral blood film. Magnetic resonance imaging (MRI) of brain (Figure $3 \& 4$ ) \& magnetic resonance venography (MRV) of cerebral vessels (Figure 5A $\& 5 \mathrm{~B})$ confirmed the presence of cerebral venous sinus thrombosis involving superior sagittal sinus, right transverse sinus, confluence area \& right lateral sinus. A hypercoagulability workup performed which included homocysteine, Protein C, Protein S, Anti-thrombin III, Fibrinogen, Anti-Cardiolipin, and Lupus screen were all negative, except for Factor $\mathrm{V}$ Leiden which was positive. D-dimer and fibrinogen degradation product (FDP) were marginally raised. Further investigations including prothrombin time, activated partial thromboplastin time (aPTT), echocardiography, carotid artery Doppler were all normal. The patient was subsequently anticoagulated with enoxaparin and warfarin. The patient made a good recovery and was subsequently discharged home 10 days post admission. A follow-up MRI and MRV studies were performed 21 days after diagnosis showed normal flow of the cerebral venous sinuses.

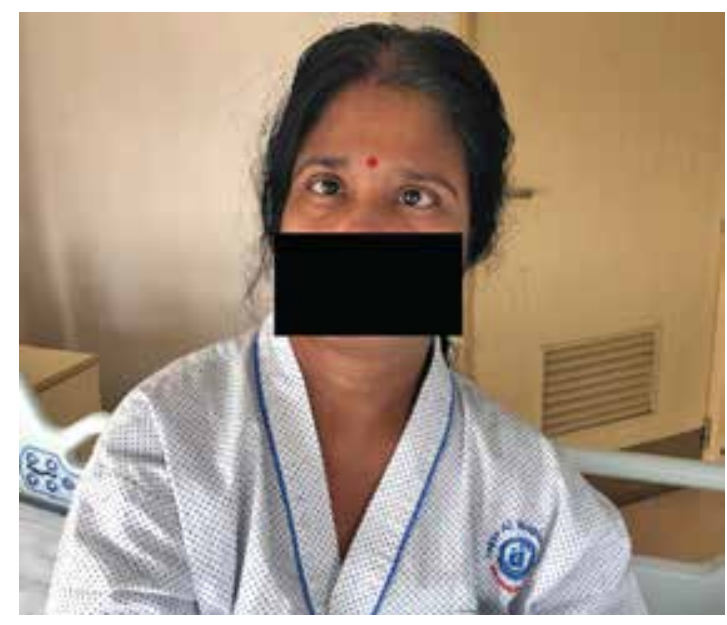

Figure 1: $\mathrm{B} / \mathrm{L} 6^{\text {th }}$ cranial nerve palsy

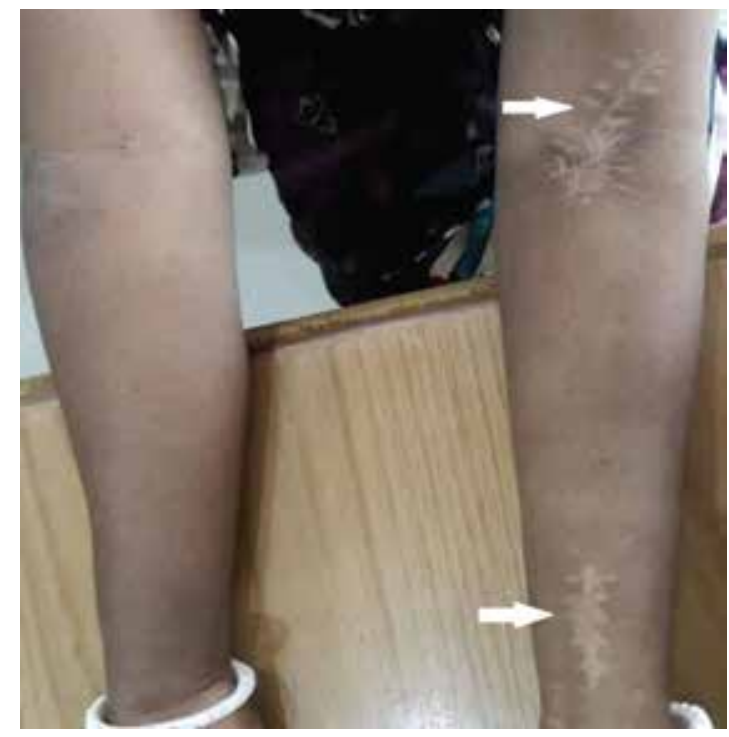

Figure 2: Embolectomy scar in left upper limb.

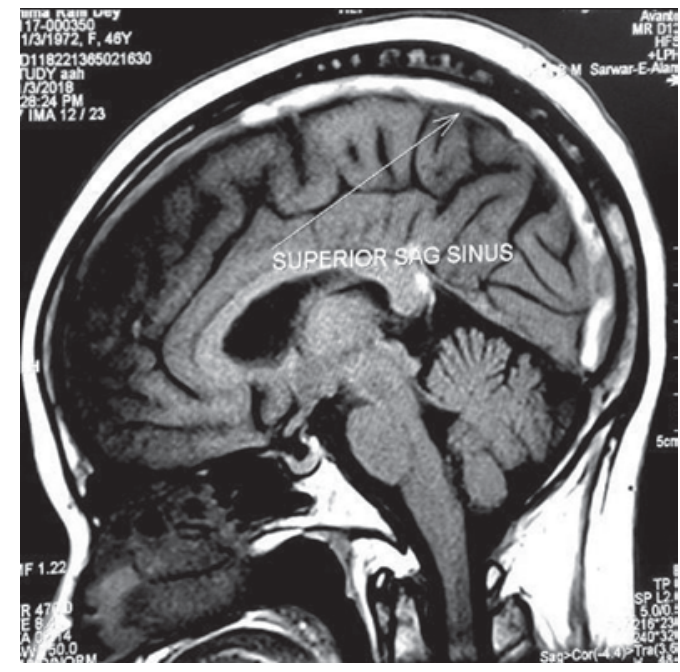

Figure 3: T1W sagittal brain MR Image without contrast showing hyperintensity of superior sagittal sinus suggestive of complete superior sagittal sinus thrombosis. 


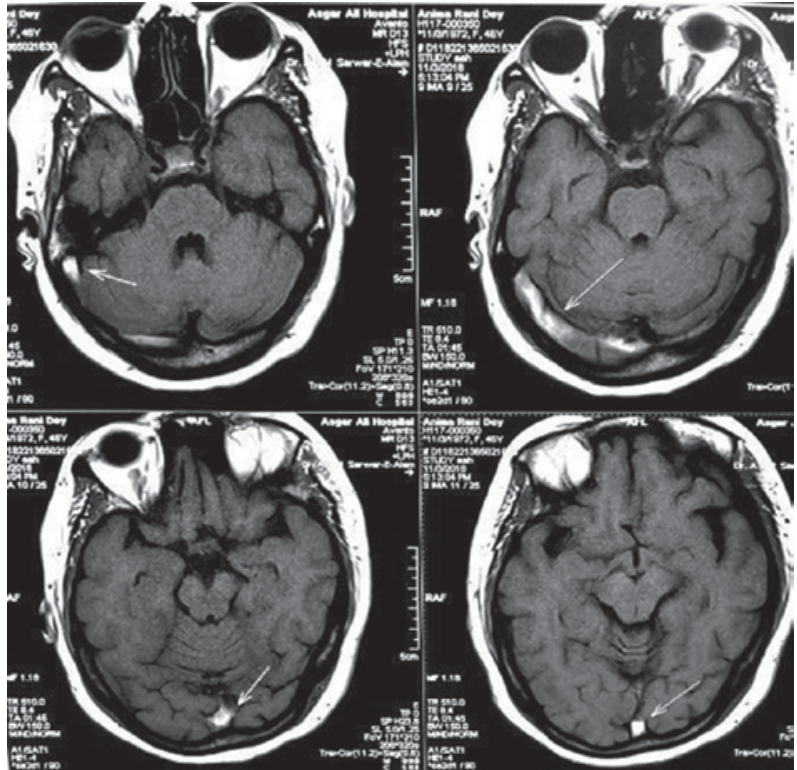

Figure 4: T1W axial brain MR Image without contrast showing hyperintensity of right transverse and right lateral sinus suggestive of right transverse and right lateral sinus thrombosis.
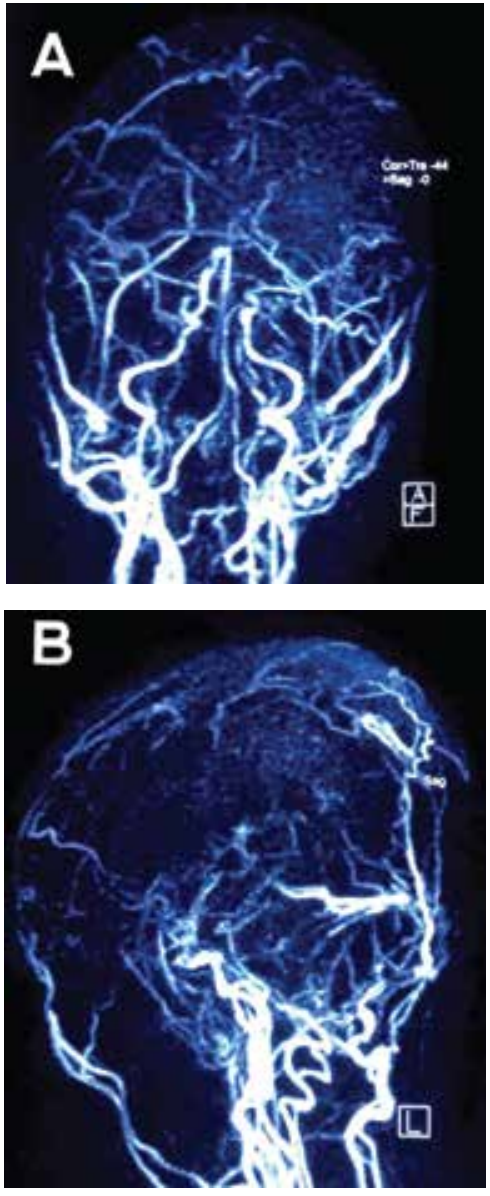

Figure 5: MR venography showing filling defect involving the superior sagittal sinus, right transverse sinus and right lateral sinus associated with few collateral veins.

\section{DISCUSSION}

Venous thromboembolism (VTE); deep venous thrombosis (DVT) and/or pulmonary embolism is a major medical problem, which affects approximately 1 in 1000 persons per year. DVT and pulmonary embolism are the most common manifestations, but thrombosis in unusual locations also occurs. VTE is a multicausal disease that results from multiple interactions between genetic, acquired, and circumstantial risk factors. Thrombophilic disorders can be identified in half of patients presenting with venous thrombosis. Inherited thrombophilia is a genetically determined tendency to develop VTE, which is often recurrent ${ }^{2}$. Factor $\mathrm{V}$ Leiden is an autosomal dominant mutation that causes activated protein $\mathrm{C}$ (APC) resistance, thus, yielding to a hypercoagulation disorder ${ }^{5}$. APC is a natural anticoagulant protein that cleaves and inactivates procoagulant Factors Va and VIIIa, thereby downregulating further thrombin generation ${ }^{2}$. Multiple genetic factors for increased thrombotic risk came with the description of APC Resistance in 1993. Dahlback et al described a large family from southern Sweden who demonstrated thrombosis in males and females throughout several generations and showed an autosomal dominant pattern of inheritance. They observed significant prolongation of the activated partial thromboplastin time (aPTT) in the normal plasma following the addition of APC. In contrast, plasma from the affected family showed a lack of significant prolongation of the APTT. The authors concluded that there was an abnormality in the protein $\mathrm{C}$ /protein $\mathrm{S}$ regulatory system $^{5}$. Subsequently, In 1994, Bertina et al at the university of Leiden first described a defect in the factor $\mathrm{V}$ gene that makes it less susceptible to inactivation by APC, this abnormality was identified as a single amino acid substitution in one of the substrate proteins for APC: Factor V ${ }^{4}$. In 1995, Kalafatis et al showed the mechanism of inactivation of the membrane bound profactor $\mathrm{Va}$ is an ordered event. Factor Va is sequentially cleaved at Arg506 and at Arg306 and Arg679 by APC. They suggested that the peptide bond cleavage at Arg506 facilitates the exposure of the subsequent cleavage sites at Arg306 and Arg679. At around the same time, Shen and Dahlback et al showed that factor $\mathrm{V}$ is also a cofactor in the inactivation of factor VIIIa by APC. The understanding of factor $\mathrm{V}$ inactivation was almost immediately followed by reports on how APC in patients' plasma failed to prolong the activated partial thromboplastin time, hence the term "APC resistance" was developed. Further studies have shown that most patients with APC resistance have a factor $\mathrm{V}$ allele that is resistant to the proteolytic effect of protein C. A transition (guanine to adenine) at nucleotide 1691 (G1691) results in the replacement of arginine by glutamine. This gene product, called factor $\mathrm{V}$ Leiden, is named after the city in the Netherlands that it was first identified in $^{3}$.

No clinical features are specific for Factor $\mathrm{V}$ Leiden thrombophilia. The diagnosis is suspected in individuals with a history of VTE especially in individuals with a personal or family history of recurrent thrombosis. The diagnosis of Factor V Leiden requires the APC resistance assay, a coagulation screening test, or DNA analysis of $F 5$, the gene 
encoding Factor V, to identify the Leiden mutation.

The APC resistance assay involves performing an activated partial thromboplastin time (aPTT) on the individual's plasma in the presence and absence of a standardized amount of exogenous APC; the two results are expressed as a ratio (aPTT $+\mathrm{APC} / \mathrm{aPTT}-\mathrm{APC}$ ). This assay is based on the principle that when added to normal plasma, APC inactivates Factors Va and VIIIa, which slows coagulation and prolongs the aPTT. The APC-resistant phenotype is characterized by a minimal prolongation of the aPTT in response to $\mathrm{APC}^{3,7}$.

Opinions and practices regarding factor $\mathrm{V}$ Leiden testing vary. There is growing consensus that testing should be performed in at least the following circumstances (these are the same general recommendations for testing for any thrombophilia):

- Age $<50$, any venous thrombosis.

- Venous thrombosis in unusual sites (such as hepatic, mesenteric, and cerebral veins).

- Recurrent venous thrombosis.

- Venous thrombosis and a strong family history of thrombotic disease.

- Venous thrombosis in pregnant women or women taking oral contraceptives.

- Relatives of individuals with venous thrombosis under age 50 .

- Myocardial infarction in female smokers under age 50.

Testing may also be considered in the following situations:

- Venous thrombosis, age $>50$, except when active malignancy is present.

- Relatives of individuals known to have factor V Leiden. Knowledge that they have factor V Leiden may influence management of pregnancy and may be a factor in decision-making regarding oral contraceptive use.

- Women with recurrent pregnancy loss or unexplained severe preeclampsia, placental abruption, intrauterine fetal growth retardation, or stillbirth. Knowledge of factor V Leiden carrier status may influence management of future pregnancies.

Random screening of the general population for factor $\mathrm{V}$ Leiden is not recommended. Routine testing is not recommended for patients with a personal or family history of arterial thrombotic disorders (e.g., acute coronary syndromes or stroke) except for the special situation of myocardial infarction in young female smokers. Neither prenatal testing nor routine newborn screening is recommended ${ }^{2,7}$.

The differential diagnosis of VTE includes other inherited and acquired thrombophilic disorders. These disorders are not clinically distinguishable, and thus laboratory testing is required for diagnosis in each case. The evaluation of high-risk individuals (i.e., those with a history of recurrent VTE, especially at a young age, or those with a strong family history of VTE at a young age) should also include assays of the following:
- Protein C activity.

- Anti-thrombin activity.

- Free Protein S antigen or Protein S activity.

The management of individuals presented with thrombosis for Factor V Leiden thrombophilia depends on the clinical circumstances. The first acute VTE should be treated with a course of low molecular weight heparin (LMWH) or intravenous unfractionated heparin. Warfarin is started concurrently with LMWH (except during pregnancy) and should be overlapped for at least 5 days and monitored with the international normalized ratio (INR) until it has been within the therapeutic range (therapeutic range: $2.0-3.0$ ) on two measurements over consecutive 2 days. INR should be targeted at 2.5, which provides effective anticoagulation, even in individuals with homozygous Factor V Leiden. Decisions regarding the duration of anticoagulation should be based on an assessment of the risks for VTE recurrence and anticoagulant-related bleeding ${ }^{8,9}$. The risk of bleeding from long-term anticoagulant therapy was greater than the benefit in patients with this mutation. But approximately $30 \%$ of patients with an incident VTE had a second event within the subsequent 9 years when not on treatment ${ }^{9}$. The optimal duration of anticoagulation for Factor V Leiden heterozygotes is debated. Factor V Leiden heterozygosity is generally not an indication for long term anticoagulation in the absence of other indications though carriers factor $\mathrm{V}$ Leiden have a higher risk of recurrence. As a consequence, individually adjusted prevention strategies following their first thrombotic episode is adopted. Recent studies suggest that prolonging anticoagulation for one year following the qualifying episode of VTE has the potential to reduce the risk of recurrent events in comparison to conventional 3-month anticoagulation ${ }^{10,11}$. Long-term anticoagulation is recommended for individuals with a first or recurrent unprovoked VTE and no risk factors for bleeding with good anticoagulation monitoring. It should also be considered in individuals with homozygous Factor $\mathrm{V}$ Leiden or with multiple thrombophilic disorders ${ }^{9,11}$. In these individuals at high risk for recurrence, new categories of oral anticoagulant drugs may be considered which have the potential to simplify the long-term treatment of patients with VTE by obviating the need for periodic laboratory monitoring, while being associated with a favourable benefit-to-risk ratio. When a thrombotic episode arises during pregnancy, it should be managed with LMWHs in full doses for at least three months, bearing in mind that the treatment should not be discontinued before the end of pregnancy, and should always be extended to cover the first six weeks after delivery ${ }^{11}$.

\section{CONCLUSION}

Thrombotic events are recognized as a significant source of mortality and morbidity. Evaluation for underlying hypercoagulable states in patients with thrombosis is encountered frequently. Factor $\mathrm{V}$ Leiden is the form of inherited thrombophilia. The clinical expression of Factor $\mathrm{V}$ Leiden thrombophilia is variable. The primary clinical 
manifestation of Factor V Leiden is VTE. Factor V Leiden is associated with a 2- to 3-fold increased risk for pregnancy loss and other complications such as preeclampsia, intrauterine growth restriction and placental abruption. Early diagnosis of Factor V Leiden reduces morbidity or mortality, decisions regarding testing should be made on an individual basis. The genetic status of asymptomatic at-risk family members can be established using molecular testing; however, heterozygosity for Factor V Leiden confers only a mildly increased risk for thrombosis, routine testing of at-risk family members is not recommended. Many individuals with a Factor V Leiden allele never develop thrombosis. Carriers of factor V Leiden have a higher risk of recurrent VTE. Anticoagulation is the mainstay of treatment of symptomatic VTE. Individuals requesting testing for Factor $\mathrm{V}$ Leiden and those identified as heterozygotes or homozygotes should be counseled on the signs and symptoms of VTE that require immediate medical attention, and the potential need for prophylactic anticoagulation in high-risk circumstances. They should be informed that although Factor V Leiden is an established risk factor, it does not predict thrombosis with certainty because the clinical course is variable even within the same family.

\section{References:}

1. Brett J Carroll, Gregory Piazza. Hypercoagulable states in arterial and venous thrombosis: When, how, and who to test? Vascular Medicine. 2018; 23(4): 388-399. DOI: 10.1177/ $1358863 X 18755927$.

2. Kujovich JL. Factor V Leiden thrombophilia. Genet Med. 2011 Jan; 13(1): 1-16. DOI: 10.1097/GIM.0b013e3181faa0f2.

3. Salwa Khan and Joseph D Dickerman. Hereditary thrombophilia. Thrombosis Journal 2006; 4:15: DOI:10.1186/1477-9560-4-15.

4. R M Bertina, B P Koeleman, T Koster, F R Rosendaal, R J Dirven, $\mathrm{H}$ de Ronde, P A van der Velden, P H Reitsma. Mutation in blood coagulation factor $\mathrm{V}$ associated with resistance to activated protein C. Nature. May 1994; 369: 64-67. DOI: 10.1038/369064a0.
5. Dahlback B, Carlsson M, Svensson PJ. Familial thrombophilia due to a previously unrecognized mechanism characterized by poor anticoagulant response to activated protein $\mathrm{C}$ : prediction of a cofactor to activated protein C. Proc Natl Acad Sci USA. Feb1993; 90(3): 1004 -1008. DOI: 10.1073/pnas.90.3.1004.

6. Rees DC, Cox M, Clegg JB. World distribution of factor V Leiden. Lancet. Oct 1995; 346: 1133-1134. DOI: 10.1016/s0140-6736(95)91803-5.

7. Grody WW, Griffin JH, Taylor AK, Korf BR, Heit JA, ACMG Factor V Leiden Working Group. American College of Medical Genetics consensus statement on factor V Leiden mutation testing. Genet Med. 2001; 3(2): 139-148. DOI: 10.1097/00125817200103000-00009.

8. Kearon C, Kahn SR, Agnelli G, Goldhaber S, Raskob GE, Comerota AJ, American College of Chest Physicians. Antithrombotic therapy for venous thromboembolic disease: American College of Chest Physicians Evidence-Based Clinical Practice Guidelines (8th Edition). Chest. 2008; 133 (6 Suppl): 454S-545S. DOI: 10.1378/chest.08-0658.

9. Baglin C, Brown K, Luddington R, Baglin T. Risk of recurrent venous thromboembolism in patients with the factor $\mathrm{V}$ Leiden (FVR506Q) mutation: effect of warfarin and prediction by precipitating factors. East Anglian Thrombophilia Study Group. Br J Haematol. March 1998; 100(4): 764 -768. DOI: 10.1046/j.1365-2141.1998.00632.x.

10. Kearon C. Natural History of Venous Thromboembolism. Circulation. Jun 2003; 107(23 Suppl 1): I-22-I-30. DOI: 10.1161/ 01.CIR.0000078464.82671.78.

11. Paolo Prandoni, Chiara Piovella, Luca Spiezia, Fabio Dalla Valle, and Raffaele Pesavento. Optimal duration of anticoagulation in patients with venous thromboembolism. Indian J Med Res. Jul 2011; 134(1): 15-21. 